\title{
Background identification and suppression for the measurement of $(n, \gamma)$ reactions with the DANCE array at LANSCE
}

\author{
R. Reifarth ${ }^{\mathrm{a}, *}$, T.A. Bredeweg ${ }^{\mathrm{a}}$, A. Alpizar-Vicente ${ }^{\mathrm{b}}$, \\ J.C. Browne ${ }^{\mathrm{a}}$, E.-I. Esch ${ }^{\mathrm{a}}$, U. Greife ${ }^{\mathrm{b}}$, R.C. Haight ${ }^{\mathrm{a}}$, \\ R. Hatarik ${ }^{\mathrm{b}}$, A. Kronenberg ${ }^{\mathrm{a}}$, J.M. O'Donnell ${ }^{\mathrm{a}}$, \\ R.S. Rundberg ${ }^{\text {a }}$, J.L. Ullmann ${ }^{\text {a }}$, D.J. Vieira ${ }^{\text {a }}$, J.B. Wilhelmy ${ }^{\text {a }}$, \\ J.M. Wouters ${ }^{\text {a }}$ \\ ${ }^{a}$ Los Alamos National Laboratory, Los Alamos, New Mexico, 87545, USA \\ ${ }^{\mathrm{b}}$ Colorado School of Mines, Golden, Colorado, 80401, USA
}

\begin{abstract}
In the commissioning phase of the DANCE project (Detector for Advanced Neutron Capture Experiments) measurements have been performed with special emphasis on the identification and suppression of possible backgrounds for the planned $(\mathrm{n}, \gamma)$ experiments. This report describes several background sources, observed in the experiment or anticipated from simulations, which will need to be suppressed in this and in similar detectors that are planned at other facilities. First successes are documented in the suppression of background from scattered neutrons captured in the detector as well as from the internal radiation. Experimental results and simulations using the GEANT code are compared.
\end{abstract}

Key words: keV neutron capture, spallation neutron source, calorimetric measurement

PACS: 28.20.Fc, 29.40.Vj, 29.25.Dz, 29.40.Wk, 25.40.Sc

\section{Introduction}

DANCE (Detector for Advanced Neutron Capture Experiments) is a $4 \pi$ detector array that consists of up to 160 elements of barium fluoride crystals.

* Corresponding author:

Email address: reifarth@lanl.gov (R. Reifarth). 
It is designed to study capture reactions on small quantities of radioactive isotopes, which are of interest to studies in nuclear astrophysics and stockpile stewardship science. DANCE is located on the $20 \mathrm{~m}$ neutron flight path 14 (FP14) at the Manuel Lujan Jr. Neutron Scattering Center at the Los Alamos Neutron Science Center (LANSCE) [1]. The neutrons are produced via spallation reactions caused by an $800 \mathrm{MeV}$ proton beam hitting a tungsten target with typical beam currents of $100 \mu \mathrm{A}$. Depending on the flight path, the fast neutrons are moderated by water or other moderators. FP14 is designed to view only the uncoupled water moderator and not the tungsten target itself. A direct neutron capture measurement in an environment like this faces three major types of background:

1. Time-independent background, which is not correlated with the neutron beam. Natural radioactivity or, in case of $\mathrm{BaF}_{2}$ detectors, intrinsic radioactivity are prominent examples. If the sample is radioactive, it will introduce another source of time-independent background.

2. Time-dependent background, which is every background component that is correlated with the time structure of the beam, but does not scale with the size of the sample in the beam. Examples are neutrons scattered from other experiments, or any particles originating at the neutron production area.

3. Sample-related background due to two processes:

3a) $(n, n)$ reactions, where the neutrons are eventually captured in the surrounding material (in case of DANCE mainly $\mathrm{BaF}_{2}$ ) and create a similar signature as capture in the sample.

3b) $(\gamma, \mathrm{X})$, where $\mathrm{X}$ includes all the possible interaction mechanisms between matter and photons (photo-effect, Compton scattering, pair production etc.). Most of the interactions are with the electrons in the sample material.

While (1) and (2) are usually determined by simple beam on/off and sample in/out experiments, more sophisticated measurements are needed to determine the sample related background (3). In order to determine the background due to scattered neutrons (3a), either the time structure or the energy information of the total $\gamma$-ray energy released is used. Classical $(n, \gamma)$ experiments were optimized to have detectors with very low neutron sensitivity (e.g. $\left.\mathrm{C}_{6} \mathrm{D}_{6}\right)$ and could only measure the resonant part of the cross section. All the events, which appeared at a time of flight between resonances were considered to be background. The neutron scatter background was not measured, because it was assumed to be negligible. However, as it turned out, this method works fine only if the capture-to-scatter cross section ratio is not too small and the required uncertainties are on the order of 10\%. For higher accuracies or less favorable cases, a better method is to detect all the $\gamma$-rays emitted during an event [2]. This means using detector material with higher gamma as well as neutron sensitivity. The number of events due to captures of scattered neutrons in the detection system will therefore be increased. But this method has the advantage that neutron captures on different isotopes, in particular on the 
sample and detector/structure materials, can be discriminated on the basis of the total energy released, or the so-called Q-value. Further background reduction might be necessary because of an unfavorable signal to background ratio. Applying appropriate cuts on total energy and multiplicity can therefore lead to a much improved signal-to-background ratio. Additionally, the significantly higher detection efficiency of such setups allows the use of smaller samples.

Background due to $\gamma$-rays (3b), which are produced at the neutron production site or from neutron capture on beam line components, can arrive at the sample position at the same time of flight as the neutrons under investigation. Without a sample in the beam, these gammas would not be detected, but since they interact with the sample (mainly via Compton-scattering and pair production), secondary gammas will be detected. Obviously this background scales with the sample size. Most of the $\gamma$-rays produced have energies below $3 \mathrm{MeV}$ and the energy deposition in a calorimetric detection system will therefore be much smaller than for the reactions under investigation.

Additionally, events due to scattered high-energy neutrons at earlier times might appear during the TOF-window under investigation, since most of the neutrons will be moderated before the final capture. Disentangling between (3a) and $(3 b)$ is therefore a challenge for modern high accuracy $(n, \gamma)$ experiments. This report describes the attempts of the DANCE collaboration to identify and suppress different background components during the different commissioning phases.

\section{Experimental Setup}

DANCE is designed as a high efficiency, highly segmented $4 \pi \mathrm{BaF}_{2}$ detector for calorimetrically detecting $\gamma$-rays following a neutron capture. The initial design work is described in [3]. For practical reasons the detector modules do not really cover the entire solid angle. The design of the detector is such that a full $4 \pi$ array would consist of 162 crystals of four different shapes, each shape covering the same solid angle [4]. Two of the 162 crystals are left out in order to leave space for the neutron beam pipe. Depending on the experiment, one crystal can be replaced by a sample changer mechanism, which makes it possible to exchange up to 3 samples without closing the beam shutter and breaking the vacuum of the beam pipe. Thus the full array is designed to host 159 or 160 out of 162 possible $\mathrm{BaF}_{2}$ crystals. The dimensions of the bare crystals are designed to form a $\mathrm{BaF}_{2}$ shell with an inner radius of $17 \mathrm{~cm}$ and a thickness of $15 \mathrm{~cm}$.

During the first commissioning phase of this project, from November 2002 until January 2003, only 147 crystals were installed in the array and only 
141 were actually connected. The main part of this report concentrates on the results obtained with 141 active crystals. Only Sect. 3.4.2 contains data taken with the full array of 159/160 crystals. In order to guide the commissioning process, intensive simulations using the detector simulation package GEANT 3.21 were performed [5]. Fig. 1 illustrates the conditions as they were simulated. The simulations were designed to be as close to the experimental conditions as possible. Therefore, the 6 mounted crystals that were not connected were included in the simulations as passive detectors, but were not drawn in the figure in order to increase the visibility of the interior of the ball. Four supporting structure pieces were also included. These pieces are welded onto the cross of the beam line inside the ball in order to increase the mechanical stability (see top left part of Fig. 1). The DANCE array is divided into two halves, left-hand side and right-hand side relative to the neutron flight direction. The position of almost all of the crystals, which were not mounted at that time, was on the equatorial ring joining the two halves. The response of the array to neutrons and gamma rays was studied using the Monte Carlo code GEANT 3.21 including the GCALOR package for low energy neutron transportation [5, 6]. The inner radius of a closed sphere with bare crystals would be $17 \mathrm{~cm}$ and the crystals have a length of $15 \mathrm{~cm}$. The crystals are supported by a spherical structure made of aluminum with an inner radius of $49.7 \mathrm{~cm}$ and an outer radius of $53.5 \mathrm{~cm}$ (1.5 inches thickness). Each crystal is wrapped in a PVC foil of $0.7 \mathrm{~mm}$ thickness and glued to a photo multiplier tube (PMT). In reality as well as in the simulations, the crystals had to be moved outwards by $1 \mathrm{~cm}$ in order to leave space for this wrapping. Together with the fact that only 160 crystals can be used, the actual solid angle covered by the detector is reduced to about $3.6 \pi$. The crystal-PMT unit was put into an aluminum housing in a way that there is no aluminum between the crystals or between crystals and sample, while the PMT is surrounded by an aluminum-housing, which was finally mounted to the surrounding supporting structure. The supporting structure as well as the aluminum housings were included in the simulations. Due to restrictions in the number of defined volumes only a simplified PMT could be included. The simplification was that the material of the PMT (mainly Co and Ni contained in the magnetic shielding) was mixed into the material of the Al-housings. In order to make the simulations as realistic as possible, the beam pipe including the cross for the sample changer were included in the simulations.

Previous GEANT simulations [3] have predicted that the background due to neutrons, which are scattered at the sample and eventually captured in the surrounding material, can be significantly reduced by a spherical ${ }^{6} \mathrm{LiH}$ shell between the sample and the $\mathrm{BaF}_{2}$ detectors. Some measurements carried out during the commissioning phase had such a ${ }^{6} \mathrm{LiH}$ moderator with an inner radius of $10.5 \mathrm{~cm}$ and an outer radius of $16.5 \mathrm{~cm}$ surrounding the target location. Therefore, most of the simulations described here were done both with and without such a moderator. The simulated ${ }^{6} \mathrm{LiH}$ absorber had a density of 

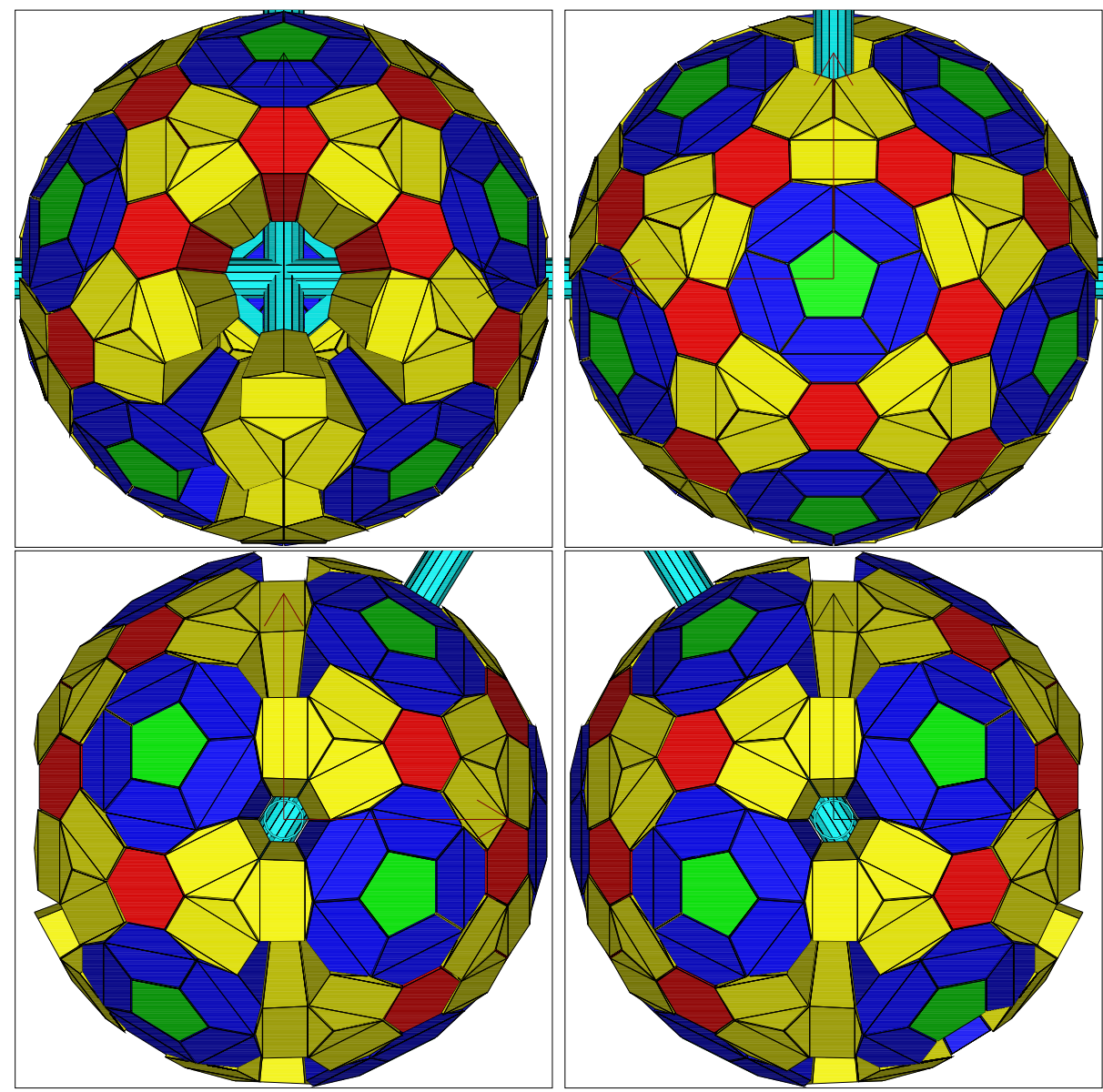

Fig. 1. Views of the simulated setup: from left / right / downstream / upstream (relative to beam direction; clockwise starting from top left). Each color corresponds to a different crystal type (A - green, B - dark blue, C - yellow, D - red). The missing crystals allowing the beam pipe (light blue) to be seen were either not connected or not in place during the runs carried out during the commissioning phase 2002/2003.

$0.85 \mathrm{~g} / \mathrm{cm}^{3}$ and a isotopic composition of $100 \%{ }^{6} \mathrm{Li}$.

In order to check the effect of scattered neutrons, a set of simulations including the walls of the detector cave (made of borated polyethylene (5\%)) and aluminum windows up- and downstream of the DANCE array has been carried out (see Section 3.3). The threshold behavior, the energy resolution and the air gap between the crystals were included in the simulations in an attempt to fit the measured spectra for various calibration source spectra. 


\section{Background components}

\subsection{Count rate estimate}

In this section a rough estimate of the expected count rate during a typical experiment will be presented. The neutron production target in the Lujan Center is typically irradiated by a pulsed $800 \mathrm{MeV}$ proton beam with an average current of $100 \mu \mathrm{A}$ and a repetition rate of $20 \mathrm{~Hz}$. Unless otherwise stated this section and all of the figures in the following sections assume a proton pulse of these specifications. Under these conditions the neutron flux at the sample position inside DANCE follows approximately a $1 / E$ law in the neutron energy region of interest $1 \mathrm{eV}<E<1 \mathrm{MeV}$. This results in a constant number of about $3 \cdot 10^{5} \mathrm{~s}^{-1}$ neutrons per energy decade, or $1.5 \cdot 10^{4}$ neutrons per energy decade per proton pulse.

Assuming a $1 / \sqrt{E}$ energy dependence for the neutron capture cross section, the neutron capture rate in the sample is expected to be constant over the duration of the neutron pulse. If the $(\mathrm{n}, \gamma)$ cross section is $\sigma(E)=c / \sqrt{E}$ and $N_{\text {sample }}$ is the number of sample atoms, the instantaneous neutron capture rate at any time during the duration of the neutron pulse would be:

$$
\begin{aligned}
\frac{\mathrm{d} C}{\mathrm{~d} t} & =\frac{\mathrm{d} \Phi}{\mathrm{d} E} \cdot \frac{\mathrm{d} E}{\mathrm{~d} t} \cdot \sigma(E) \cdot N_{\text {sample }} \\
& =2.7 \cdot 10^{8} \cdot c \cdot N_{\text {sample }} \frac{1}{\sqrt{\mathrm{keV}} \mathrm{s} \mathrm{cm}^{2}}
\end{aligned}
$$

Inserting typical values of $c=1 \mathrm{barn} \sqrt{\mathrm{keV}}$ and $N_{\text {sample }}=6 \cdot 10^{18}(1 \mathrm{mg}$ with atomic mass 100) gives $1.65 \cdot 10^{3}$ neutron capture per second, which will be detected with nearly $100 \%$ efficiency. Most of the neutron capture events will cause a signal with summed $\gamma$-energy of $\pm 500 \mathrm{keV}$ around the Q-value of the reaction. Based on these assumptions an instantaneous capture rate of $10^{3} \mathrm{~s}^{-1}$ distributed over a $\gamma$-energy range of $1 \mathrm{MeV}$ will be used for comparison with the achieved background levels in later sections.

\subsection{Time-independent background}

Time-independent background is not correlated with the time structure of the neutron beam. In our case natural radioactivity, the intrinsic radioactivity of the $\mathrm{BaF}_{2}$ detectors and that of a radioactive sample have to be considered.

Natural $\gamma$-activity, like ${ }^{40} \mathrm{~K}$ and ${ }^{208} \mathrm{Tl}$, with $\gamma$-ray energies of $1465 \mathrm{keV}$ and 
$2615 \mathrm{keV}$, respectively, can be discriminated from neutron capture events using information about the gamma energy deposited in the detector. The neutron binding energy is above $5 \mathrm{MeV}$ for most of the isotopes under investigation, which is well separated from the typical $\gamma$-ray energies of natural decays. This means that most of the events due to neutron capture on the sample appear at much higher channels in the sum energy spectrum. As it will become clearer in later sections, it is still desirable to understand and - ideally - reduce or discriminate those lower-energy background components by other means than the energy information. The main reason for that is the fact that DANCE does not have a $100 \%$ detection efficiency for $\gamma$-rays and consequently a significant part of the $(\mathrm{n}, \gamma)$ events deposit therefore less energy than the Q-value. For high accuracy measurements, this low energy tail of the energy distribution needs to be well understood and, if possible, measured with low background.

The intrinsic radioactivity of the $\mathrm{BaF}_{2}$ crystals originates from the $\alpha$-decay chain of the chemical homologue ${ }^{226} \mathrm{Ra}$, see Fig. 2. Typical activities are $0.2 \mathrm{~Bq} / \mathrm{cm}^{3}$ material [2]. Most of the crystals used for DANCE show an intrinsic $\alpha$-activity between 150 and $250 \mathrm{~Hz}$. The scintillation light of $\mathrm{BaF}_{2}$ crystals has a short $(\approx 0.6 \mathrm{~ns})$ and a long $(\approx 0.6 \mu \mathrm{s})$ component. The intensity ratio between the two components is radiation type dependent, which allows for particle identification if both components are measured. While the emitted $\alpha$ particles deposit their energy almost exclusively through the slow component, about $10 \%$ of the energy of the $\gamma$-rays feeds the fast component. Therefore a comparison of the fast and slow component integrals provides a powerful tool to reject the background due to $\alpha$-activity. In our experiment each $\mathrm{BaF}_{2}$ signal is recorded with waveform digitizers (Acqiris Corp., model DC-265) and integrated with two different integration times, each matching either the short or the long decay time. Fig. 3 shows the results of this method applied to a run carried out with a ${ }^{60} \mathrm{Co}$ calibration source in the center of the DANCE array. Applying appropriate cuts results in an almost complete suppression of the $\alpha$-background.

During the runs carried out at DANCE a significant background component between 0.5 and $3.5 \mathrm{MeV}$ was discovered, which could not be discriminated via particle identification (see Fig. 4). The observed count rate per crystal is about the same as the $\alpha$-activity. As a result of the ${ }^{226} \mathrm{Ra}$ inside the $\mathrm{BaF}_{2}$, the crystals contain not only $\alpha$, but also $\beta^{-}$activities. The most important contributions are coming from ${ }^{214} \mathrm{Bi}\left(\beta^{-}\right)\left(Q_{\beta}=3.3 \mathrm{MeV}\right)$ and ${ }^{214} \mathrm{~Pb}\left(\beta^{-}\right)\left(Q_{\beta}=1.0 \mathrm{MeV}\right)$. All other decays either release energies below $100 \mathrm{keV}$ or are not yet in equilibrium, since ${ }^{210} \mathrm{~Pb}$ with a half life of 22.3 years acts as a bottle neck in the decay chain. In order to confirm these decays as the cause of the observed background, GEANT simulations have been carried out. In a first step, decay cascades according to the (in the case of ${ }^{214} \mathrm{Bi}\left(\beta^{-}\right)$rather complex) decay scheme [7, 8] have been created using a Monte Carlo method. The energy distribution of the emitted electrons was calculated by means of the Fermi 


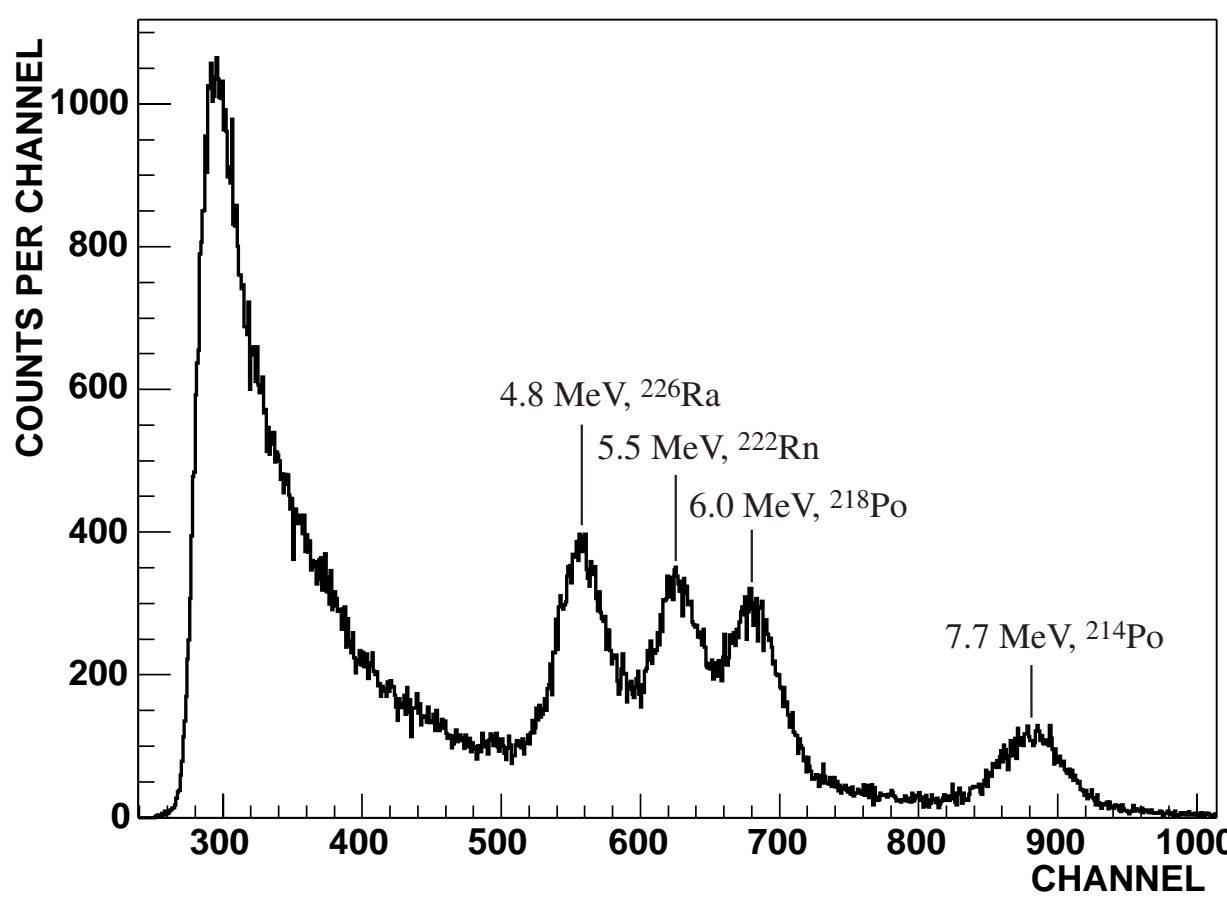

Fig. 2. The picture shows an $\alpha$-background spectrum for a single crystal without beam. The labeled peaks correspond to different $\alpha$ 's from the ${ }^{226} \mathrm{Ra}$ decay chain.

theory of beta decay. In a second step, these cascades were started inside the $\mathrm{BaF}_{2}$ scintillators and the response of the array was simulated. Fig. 5 shows the result for $10^{5}$ decays of ${ }^{214} \mathrm{Bi}$ and ${ }^{214} \mathrm{~Pb}$. The agreement between the experimental data (Fig. 4) and the corresponding spectrum in the middle section of Fig. 5 is remarkable. The bottom part of Fig. 5 shows the same data with a cluster multiplicity cut of greater than 1 applied. A cluster is defined as a set of neighboring fired crystals, completely surrounded by crystals that register no hits. The simulations suggest that this background can be significantly discriminated using such a cluster analysis. While the total detection efficiency for a ${ }^{214} \mathrm{Bi}$ or ${ }^{214} \mathrm{~Pb}$ event is above $99 \%$, the probability for events creating more than 1 cluster is $16 \%$ or $3 \%$, respectively. For typical neutron capture events however this would be more than $80 \%$ [9].

\subsection{Time-dependent background}

Time-dependent background is correlated with the time structure of the neutron beam, but does not scale with the size of the sample. At pulsed neutron sources the neutrons are usually preceded by other particles, e.g. the so-called $\gamma$-pulse $(\gamma$-flash), originating from the interaction of the primary beam with the neutron production target. Because FP-14 is designed to view an upper tier water moderator and not the tungsten cylinder directly, the DANCE detector is shielded against primary particles from the production target. Additionally the apertures used for neutron collimation restrict the geometrical 


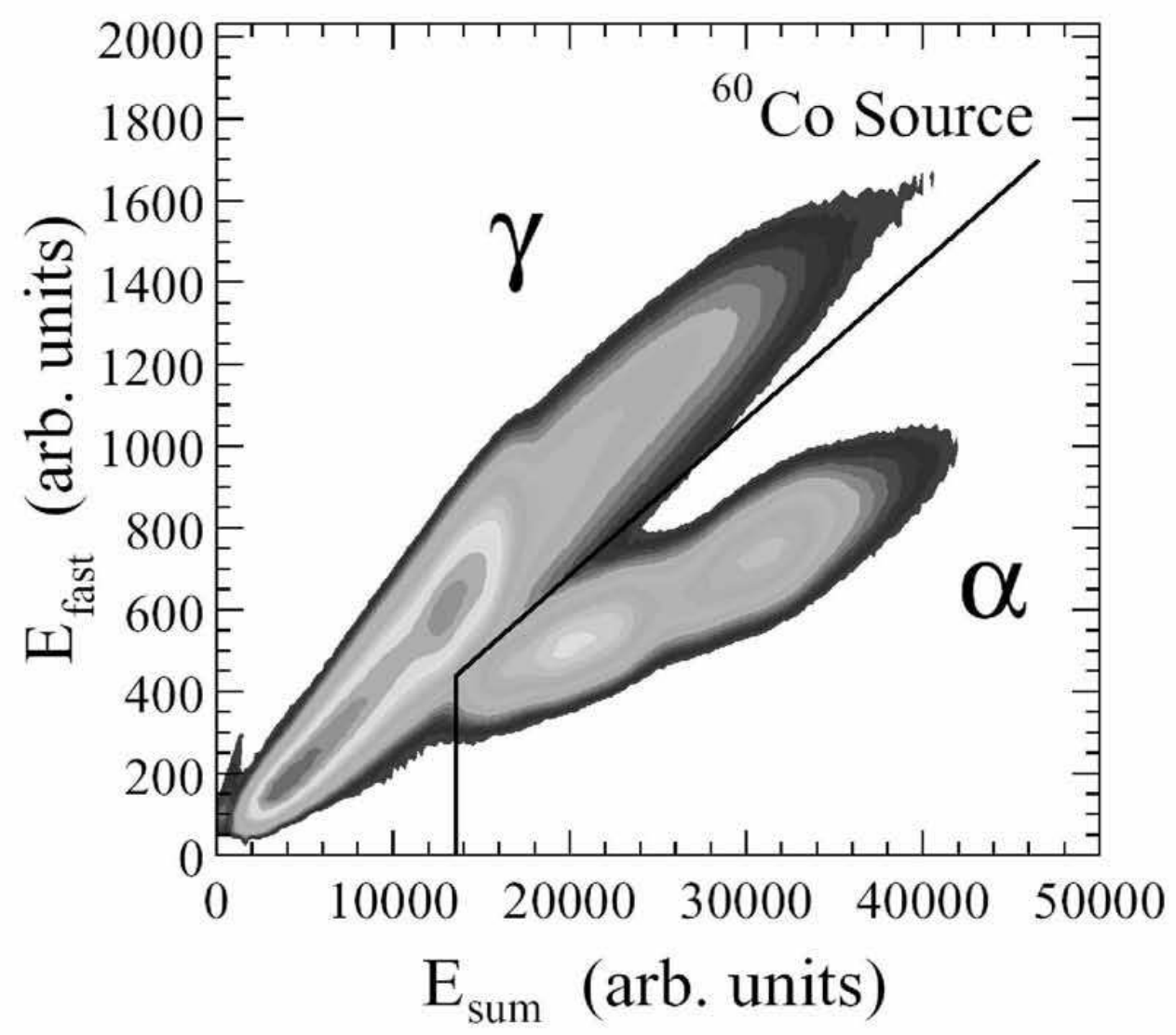

Fig. 3. A two-dimensional plot of the fast versus the sum of fast and slow component of the $\mathrm{BaF}_{2}$ signal for a ${ }^{60} \mathrm{Co}$ calibration source is shown. The lower right part is cut out in order to discriminate the intrinsic $\alpha$-background and gain an improved signal to noise ratio.

size of the flow for most of the background particles coming along the flight path. Therefore, without any material in the neutron beam almost no prompt $\gamma$-flash would be observed with the DANCE array.

Nevertheless, due to practical reasons a set of windows is installed on FP-14 in order to divide the beam pipe volume into smaller segments. Furthermore a neutron monitor is installed downstream of the DANCE array. Neutrons as well as $\gamma$-rays (the most important second component in the beam) interact with these windows and cause time-dependent background in the $\mathrm{BaF}_{2}$ detector. Fig. 6 shows the number of events per pulse as a function of time of flight without any sample in the beam.

Based on Monte Carlo simulations and the observed rather high beam induced background all the previously used, rather thick, Al-windows inside the experimental area have been replaced by KAPTON foils of $25 \mu \mathrm{m}$ (1 mil) thickness and the neutron monitor has been moved further downstream. Both changes significantly reduced the time-dependent background (see Fig. 7). 


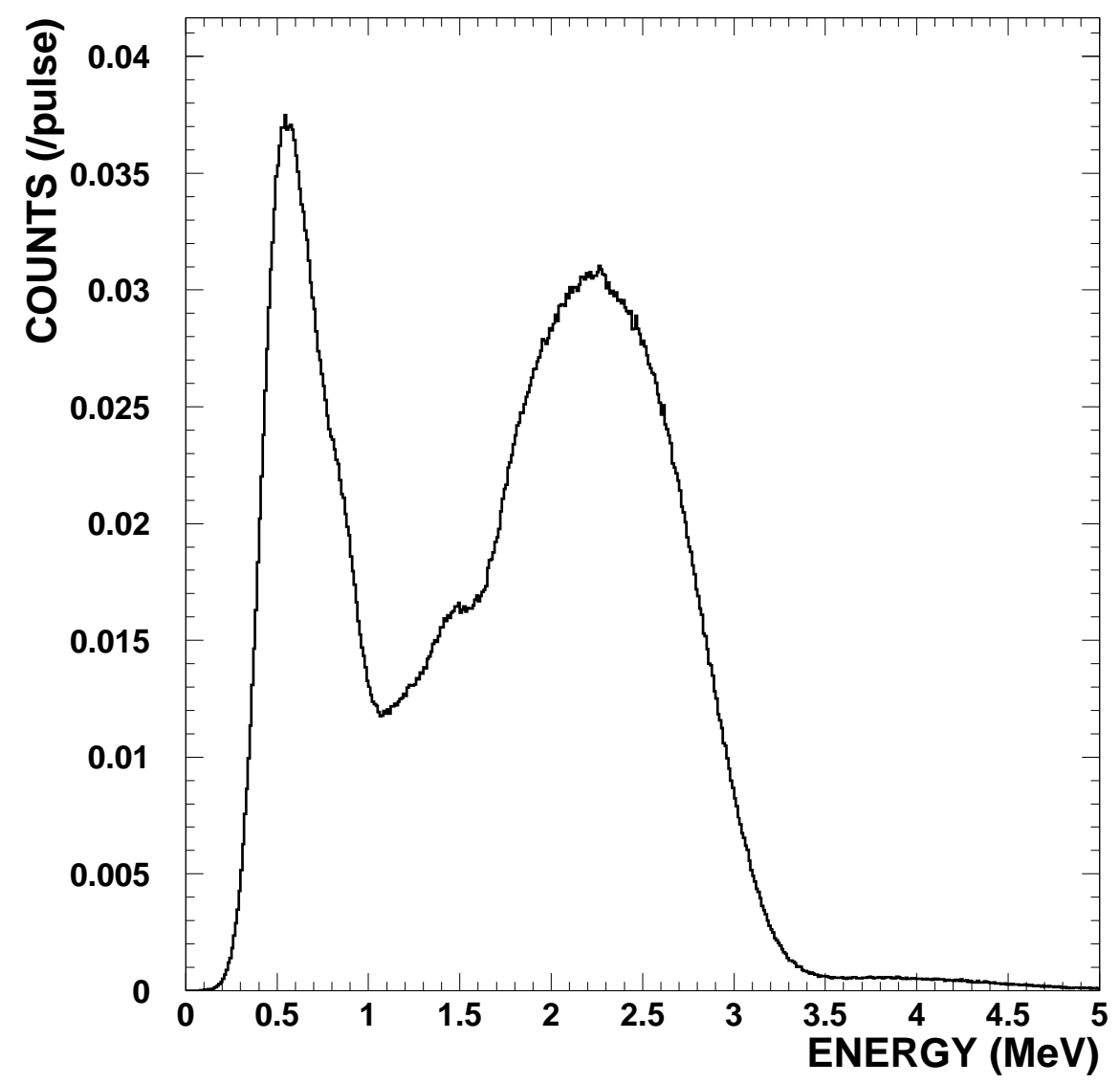

Fig. 4. Measured background for the DANCE array (160 crystals) without beam. Shown is the total energy for all events with detector multiplicity 2 or higher. The cut on multiplicity effectively eliminates events arising from internal $\alpha$-decays (e.g. Fig. 2). Histogram binning is $10 \mathrm{keV} /$ channel. One pulse corresponds to $2 \mathrm{~ms}$ looking time. The rate estimate given in Sect. 3.1 would be 0.02 in this plot.

\subsection{Sample-related background}

For the purpose of this article only background created by the interaction of particles in the beam with the sample are under investigation. The possible radioactivity of the sample is not discussed here.

\subsubsection{Neutron scattering reactions}

The scattering cross section for neutrons with keV energies is typically 12 orders of magnitude larger than the capture cross section. Therefore, this unavoidable background component might dominate the experiment. Even though the concept of a $100 \%$ efficiency detector in principle allows discrim- 


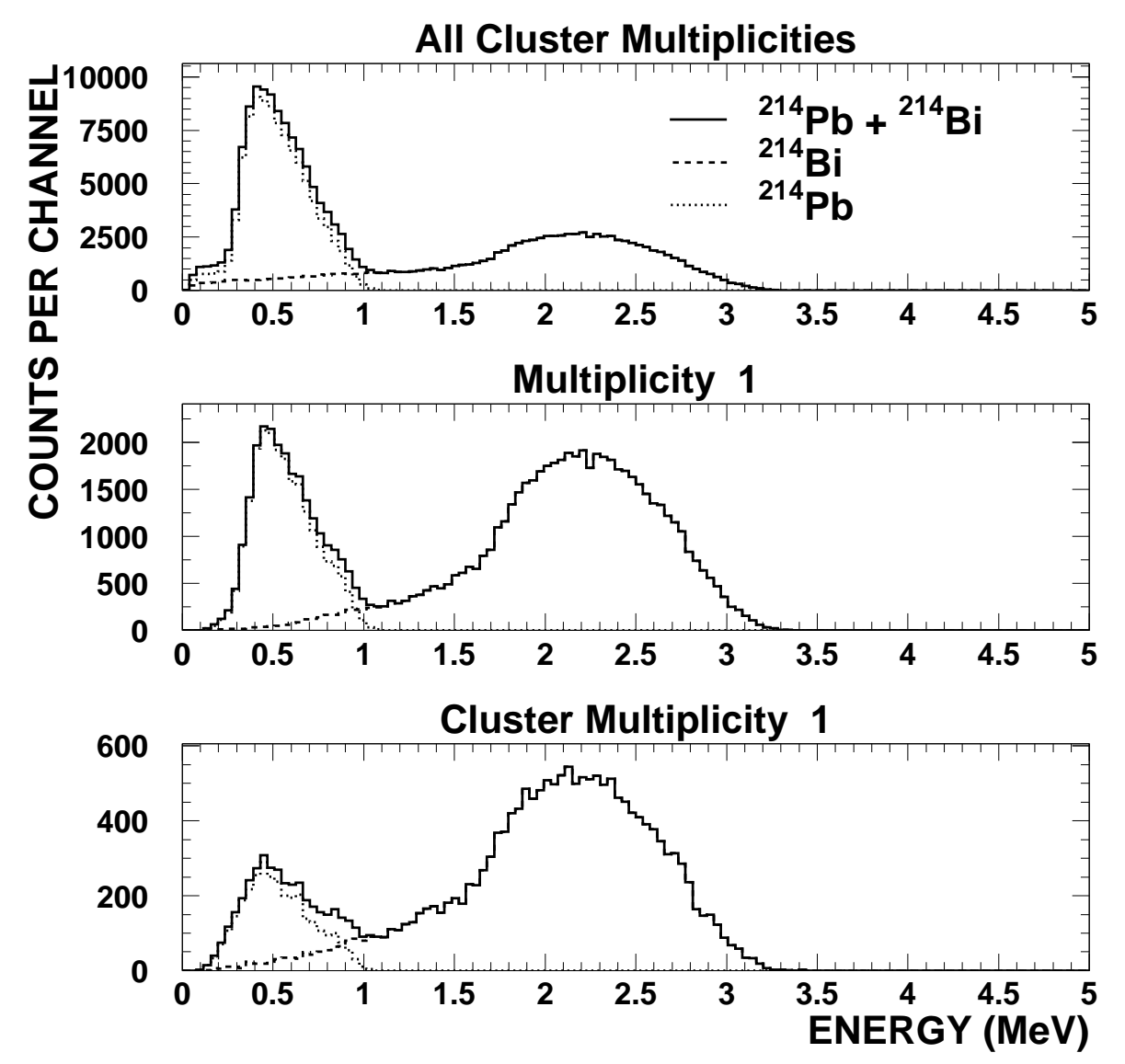

Fig. 5. Simulated background due to the decay of the two daughters ${ }^{214} \operatorname{Bi}\left(\beta^{-}\right)$and ${ }^{214} \mathrm{~Pb}\left(\beta^{-}\right)$of the ${ }^{226} \mathrm{Ra}$ decay chain for $10^{5}$ decays of each isotope. The events below $1 \mathrm{MeV}$ are mainly due to the decay of ${ }^{214} \mathrm{~Pb}$ with a $\mathrm{Q}$-value of $1.03 \mathrm{MeV}$, while all the events above $1 \mathrm{MeV}$ correspond to decays of ${ }^{214} \mathrm{Bi}$ with a Q-value of $3.27 \mathrm{MeV}$.

ination between neutron captures on different isotopes, further reduction of background due to scattered neutrons is necessary. The significance of this problem has been explored by means of Monte-Carlo simulations of neutrons on a gold sample using GEANT 3.21 [10], as shown in Fig. 8.

The DANCE collaboration decided to use a passive method of reducing the number of neutrons scattered into the surrounding materials. A ${ }^{6} \mathrm{LiH}$ shell with an inner radius of $10.5 \mathrm{~cm}$ and an outer radius of $16.5 \mathrm{~cm}$ was installed inside the DANCE ball. This shell efficiently moderates and absorbs neutrons scattered at the sample without producing $\gamma$-rays (see Fig. 9 and Table 1). Furthermore, the material has been chosen to be as transparent as possible for gamma radiation originating from neutron captures on the sample. Nonetheless, the simulations predicted a reduction of the sum peak efficiency (Fig. 10). The experimental effect of the ${ }^{6} \mathrm{LiH}$ shell can be seen in Fig. 11, where two runs with a lead sample are compared. Natural lead predominantly scatters 


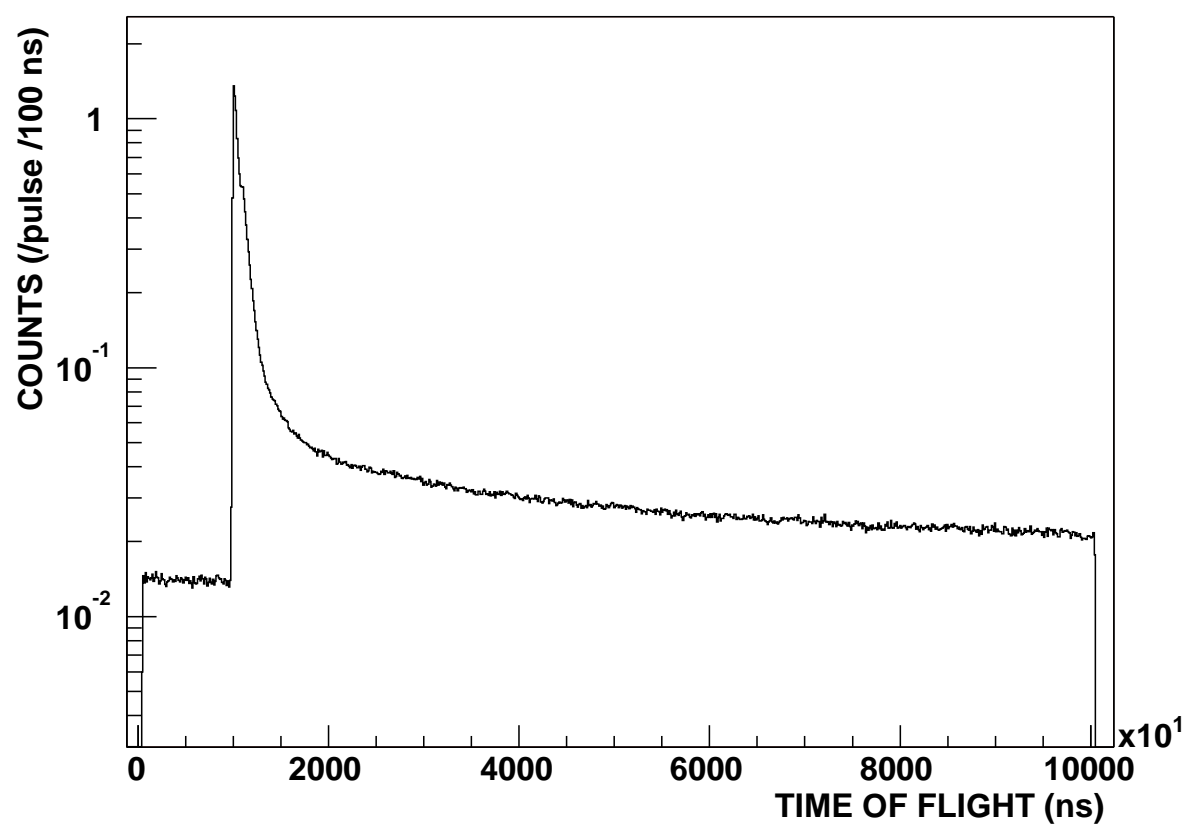

Fig. 6. Beam-related background measured at DANCE. The first $10 \mu$ s are preceding the actual proton pulse and characterize the time-independent background. No multiplicity or energy cut has been applied. The right end of the TOF spectrum corresponds to a neutron energy of $260 \mathrm{eV}$. With the estimate given in Sect. 3.1, the count rate for neutron capture would be $10^{-4}$ in this plot. In this case of no energy or multiplicity cuts, the background rate is 2 orders of magnitude above the neutron capture rate.

Table 1

Ratio of events from scattered neutrons and capture events on the sample for different setups and neutron energy intervals. The simulations contained $141 \mathrm{BaF}_{2}$ crystals and a high detector threshold. The last column is for events with total deposited energy above $1 \mathrm{MeV}$ only, while all other ratios correspond to the total number of detected events. The last line shows the ratio between the two first lines.

\begin{tabular}{lcccc} 
Setup & $0.1 \ldots 1 \mathrm{keV}$ & $1 . .10 \mathrm{keV}$ & $10 . .100 \mathrm{keV}$ & $0.1 . .1 \mathrm{MeV}$ \\
\hline no ${ }^{6} \mathrm{LiH}$ & 0.47 & 1.2 & 2.2 & 2.7 \\
${ }^{6} \mathrm{LiH}$ & 0.0035 & 0.025 & 0.20 & 0.66 \\
Ratio & 134 & 48 & 11 & 4.1
\end{tabular}

neutrons. The peaks due to resonant capture of scattered neutrons on the surrounding barium are at least a factor of 20 reduced after installing the ${ }^{6} \mathrm{LiH}$ shell, while the peaks due to resonant captures on the Sb impurities inside the lead sample remain at the same level. 


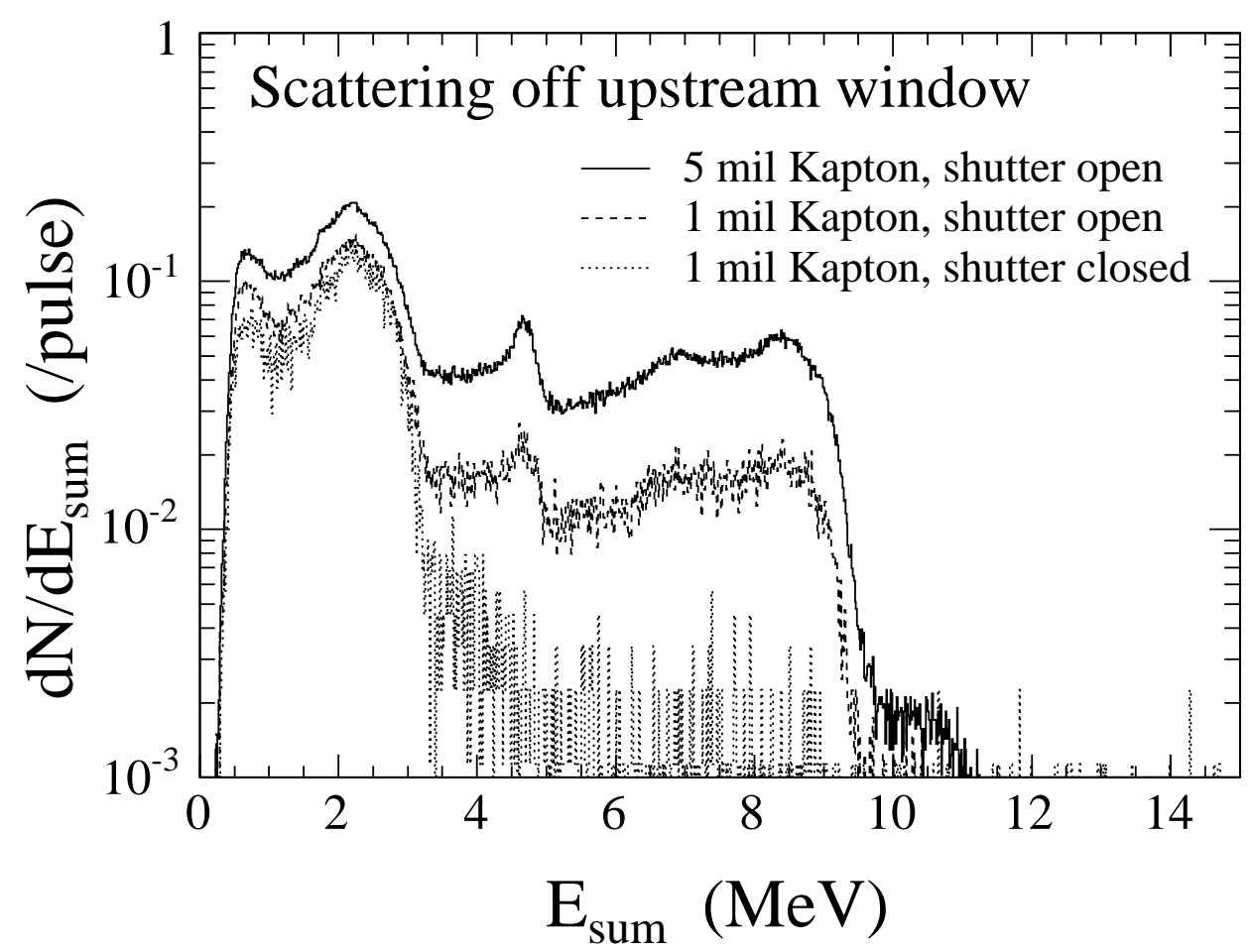

Fig. 7. Example for background reduction achieved by optimizing beam pipe windows. Histogram binning is $15 \mathrm{keV} /$ channel. One pulse corresponds to $1.6 \mathrm{~ms}$ looking time. The rate estimate given in Sect. 3.1 is $2.4 \cdot 10^{-2}$ for this plot, which is above the background rate for energies of $3 \mathrm{MeV}$ or higher. This shows that the discussed background reduction was sufficient.

\subsection{2 $\gamma$-induced reactions}

Experiments and FLUKA simulations at n-TOF as well as GEANT and MCNP simulations for target 1 at WNR show that about $1 \gamma$-ray per 10 neutrons will be present at the sample position in the keV-neutron TOF region. The detection efficiency of the DANCE array for photons is about $90 \%$, while it is only $10 \%$ for scattered neutrons (without the ${ }^{6} \mathrm{LiH}$ absorber shell). Thus the potential ratio of events caused by scattered neutrons to events by scattered photons is about equal, before weighting with the respective cross sections due to interaction with the isotope at the sample position.

The $\gamma$-ray spectrum depends strongly on the reaction producing the photons as well as on the specifics of the setup. The $\gamma$-spectrum at late times (long time of flight) consists mainly of two components:

- prompt photons due to capture of moderated neutrons

- photons due to the decay of (neutron induced) reaction products

The component due to the decay of reaction products was measured at FP14 with a germanium detector three months after the shutdown of the accelerator (see Fig. 12). All the observed lines correspond to activation due to neutron 


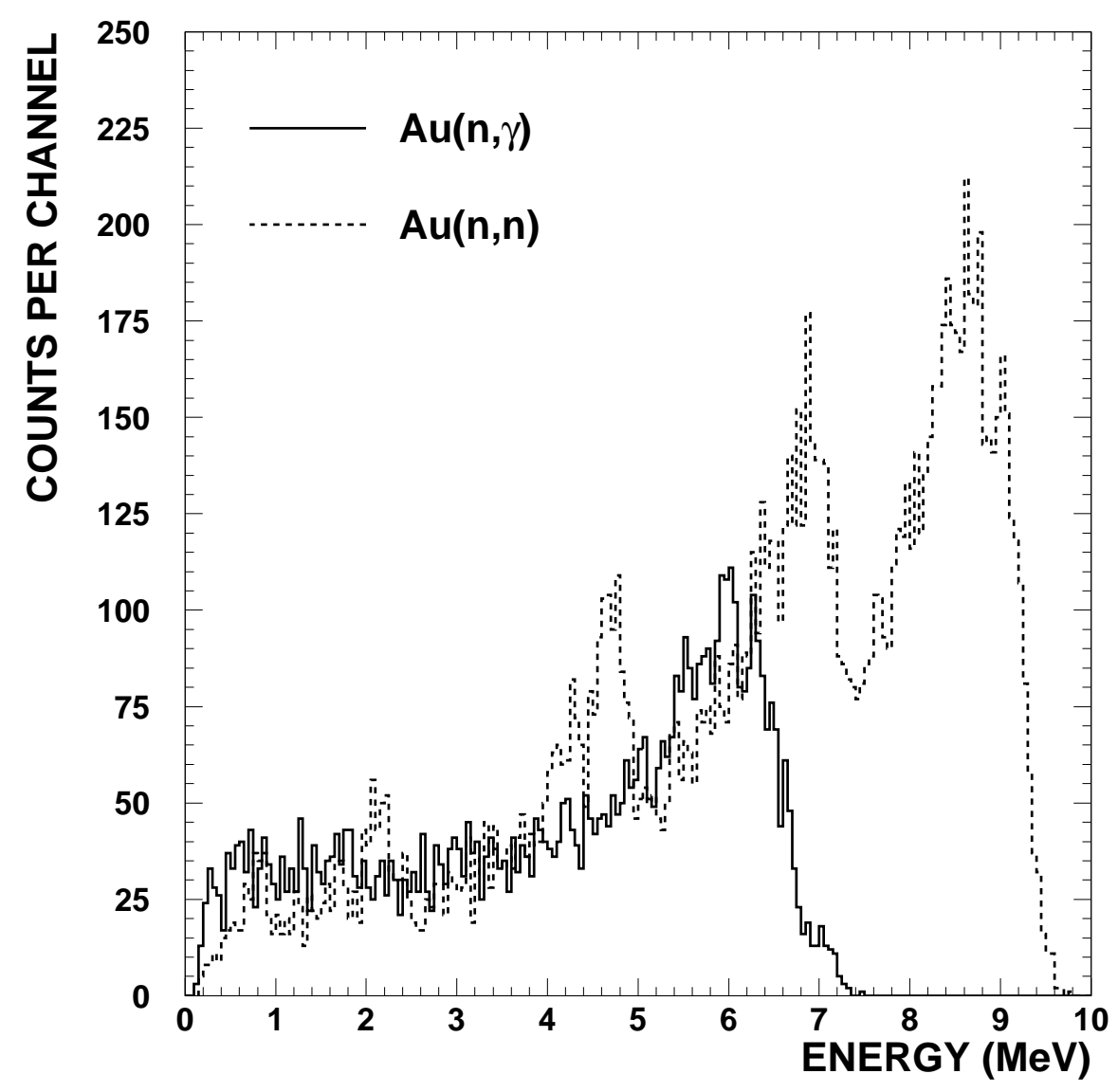

Fig. 8. Simulated total energy spectra for the DANCE array with 141 crystals and high single detector threshold without the ${ }^{6} \mathrm{LiH}$ absorber. $10^{7}$ neutrons between $10 \mathrm{keV}$ and $100 \mathrm{keV}$ have been emitted towards a $0.2 \mathrm{~mm}$ thick gold sample. The solid line corresponds to events due to captures on a gold sample, the dashed curve to events due to neutrons scattered at the gold sample and captured on the isotopes of $\mathrm{Ba}$ and $\mathrm{F}$. Histogram binning is $50 \mathrm{keV} /$ channel.

induced reactions on aluminum alloy and stainless steel. Both materials are present in windows upstream of the shutter, and the water moderator container is made from aluminum alloy.

The capture $\gamma$-ray component has not been measured directly yet. Since both neutron as well as gamma flux during accelerator operations is very high, it is almost impossible to place any kind of detector directly in the beam. Indirect methods need to be applied. Depending on the atomic number of a sample in the beam, the total interaction cross section varies significantly as a function of the photon energy. The DANCE array is a powerful tool to detect the $\gamma$-ray component of such interactions, since almost all of the secondary $\gamma$-rays will be absorbed in the $\mathrm{BaF}_{2}$ crystals. 


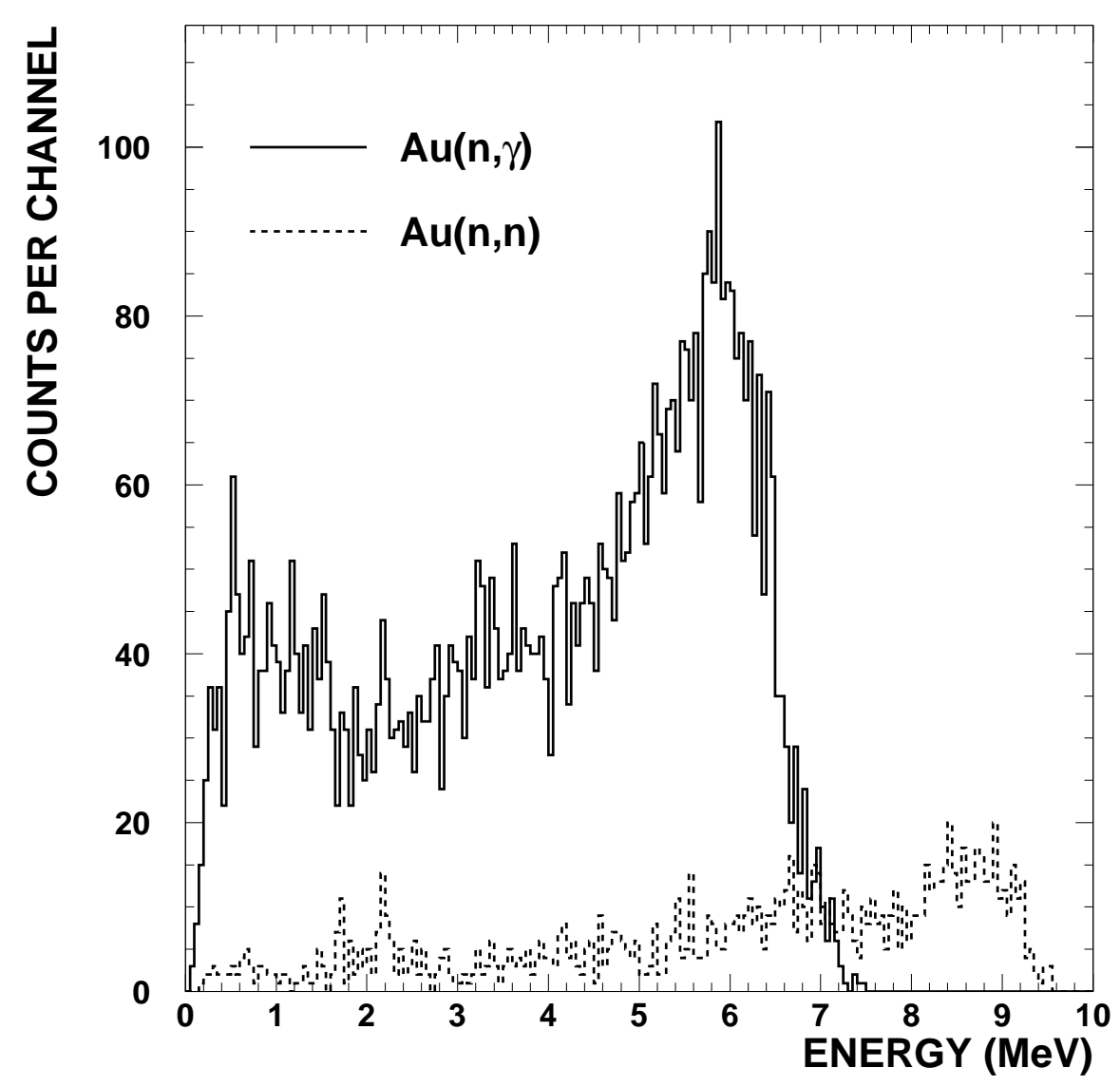

Fig. 9. Simulated total energy spectra for the DANCE array with 141 crystals and high single detector threshold with the ${ }^{6} \mathrm{LiH}$ absorber. $10^{7}$ neutrons between $10 \mathrm{keV}$ and $100 \mathrm{keV}$ have been started towards a $0.2 \mathrm{~mm}$ thick gold sample. The solid line corresponds to events due to captures on a gold sample, the dashed curve to events due to neutrons scattered at the gold sample and captured on the isotopes of $\mathrm{Ba}$ and F. Histogram binning is $50 \mathrm{keV} /$ channel.

Carbon is often used for determining the neutron scattering background, since the neutron capture cross section is orders of magnitude smaller than the scattering cross section. Fig. 13 shows a simulated response of the $4 \pi$ detector to photons on a carbon sample, typical for samples with low atomic number. The most important interaction mechanisms in this case are photo effect and Compton scattering. Only high-energy photons interact primarily due to pair production. Therefore, the $1.02 \mathrm{MeV}$ peak due to pair production can only be observed for primary photons above $4 \mathrm{MeV}$. As mentioned previously, another important neutron scattering sample is lead. In contrast to the case of carbon, the spectra gained for a lead sample reflect the typical for photon interaction with heavy nuclei (Fig. 14). Pair production becomes the most important interaction, resulting in a peak at $1.02 \mathrm{MeV}$. For more detailed information on those simulations see also Ref. [11]. 


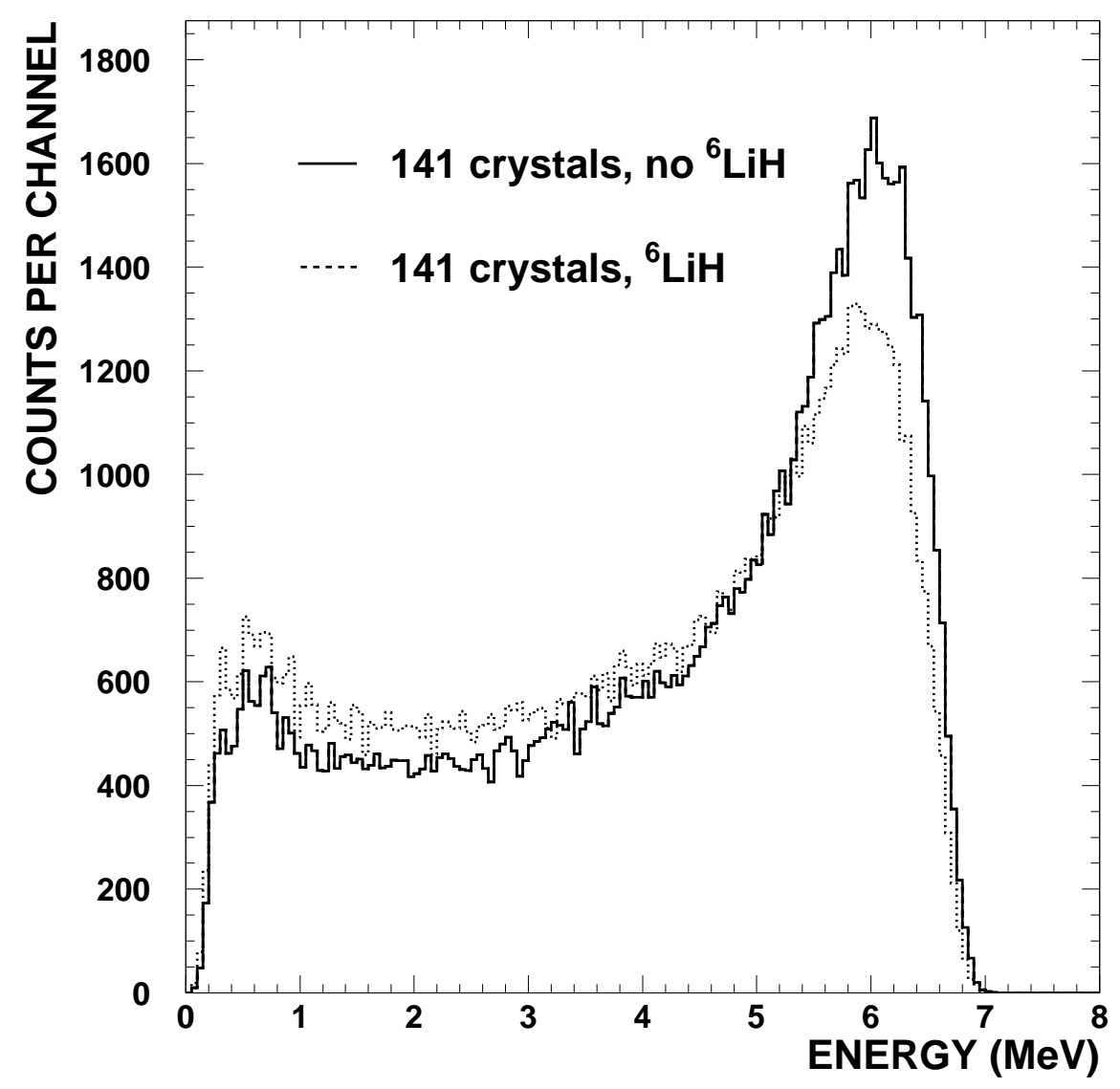

Fig. 10. Simulated total energy spectra for the DANCE array with 141 crystals and high single detector thresholds for gamma radiation from neutron capture on gold. $10^{5}$ cascades were simulated. The histogram binning is $50 \mathrm{keV} /$ channel. For details about the capture cascades used for these simulations see Ref. [12].

Figs. 15 and 16 show measured spectra, which underline the significance of this background component. For these runs a carbon and a lead sample have been placed in the beam and the spectra have been taken with the DANCE array. The TOF interval is the same as in Fig. 6, corresponding to neutron energies of $0.26 \mathrm{keV}$ or higher. A $1.3 \mathrm{~cm}$ thick absorber of polyethylene was placed approximately in the middle between the neutron production target and the sample, which reduced the total neutron flux at the sample below $100 \mathrm{keV}$ by at least a factor of 10 , while the $\gamma$-ray flux is reduced less than $5 \%$. Therefore most of the events shown in the figures are related to photon interactions with the sample. Especially in the case of lead, the peak at $1.02 \mathrm{MeV}$ can be clearly recognized. The absence of this peak in the case of carbon suggests a $\gamma$-ray spectrum with energies predominantly between the pair production threshold (since lead shows the $1.02 \mathrm{MeV}$ peak) and $3 \mathrm{MeV}$ (otherwise carbon should show the $1.02 \mathrm{MeV}$ peak too). A possible production mechanism could be $\mathrm{H}(\mathrm{n}, \gamma)$ in the moderator, resulting in a $\gamma$-ray with an energy of $2.2 \mathrm{MeV}$. 


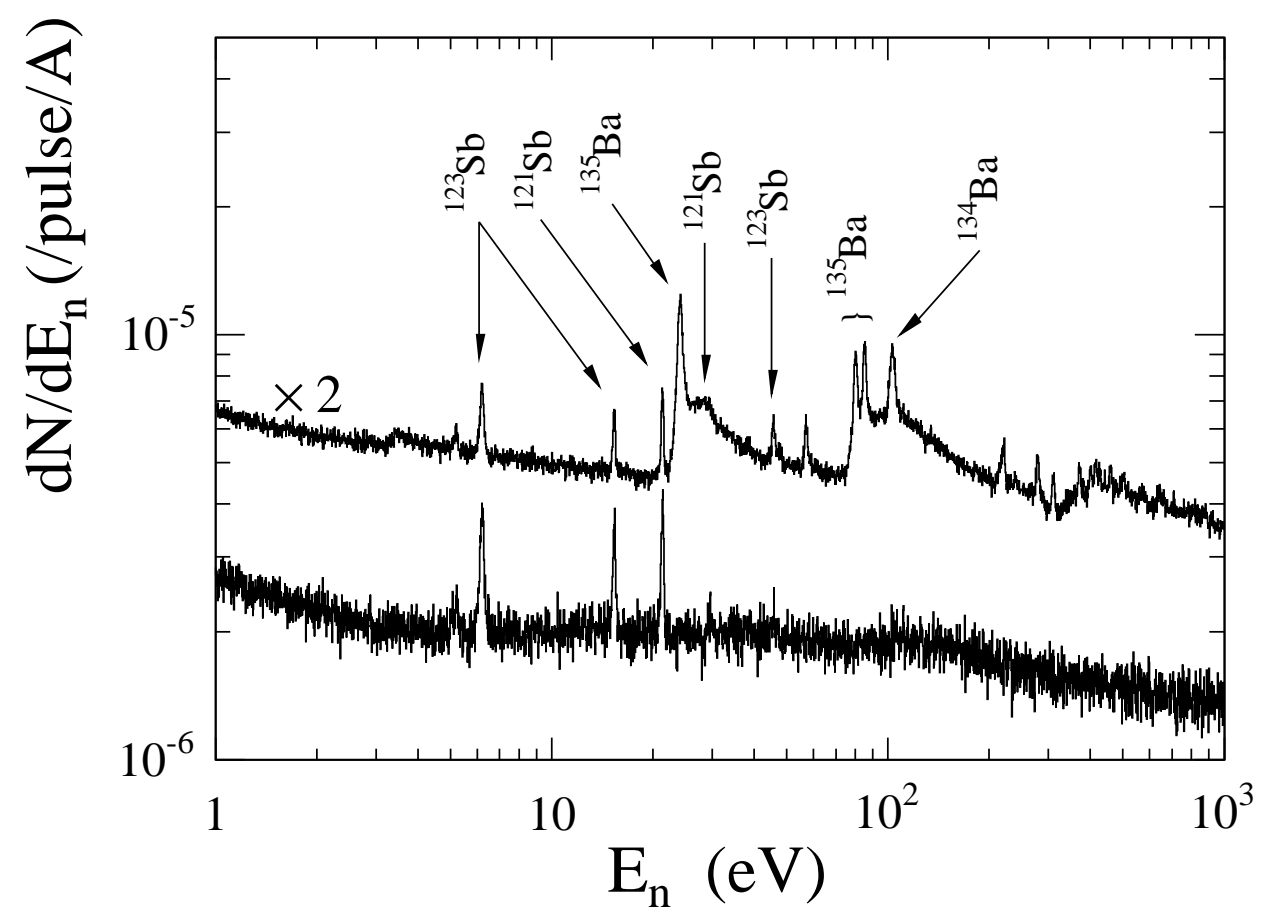

Fig. 11. Events detected with DANCE as a function of incident neutron energy. A $43.5 \mathrm{mg} / \mathrm{cm}^{2}$ lead sample was in the beam and 141 crystals were connected. Top curve: Without ${ }^{6} \mathrm{LiH}$ absorber Bottom curve: With ${ }^{6} \mathrm{LiH}$ absorber. The spectra are normalized to proton beam current (in ampere) and pulse. The binning of the x-axis is logarithmic and contains 1000 channels per energy decade. Only a single detector energy threshold of about $400 \mathrm{keV}$ was applied.

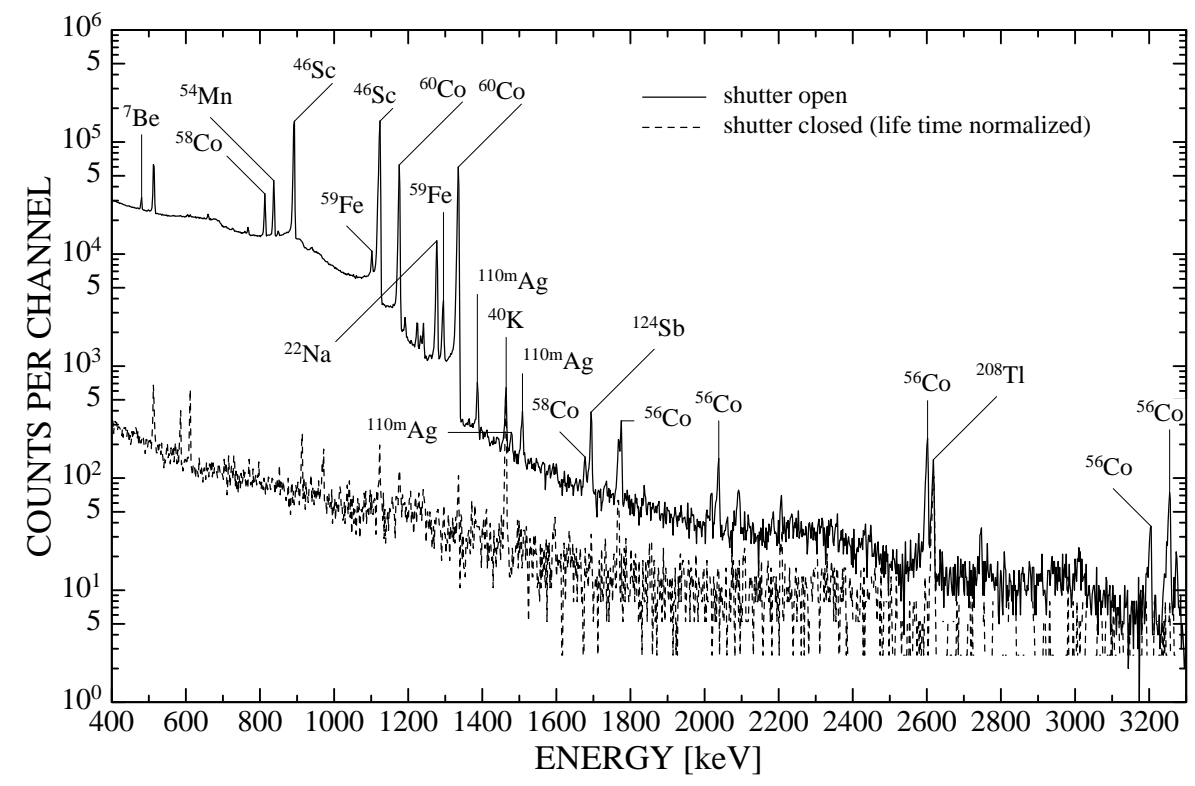

Fig. 12. Gamma-ray spectrum measured with a germanium detector at flight path 14 at the Manual Lujan Center at LANL 3 months after the last neutron beam. Both runs are normalized to the same life time. 

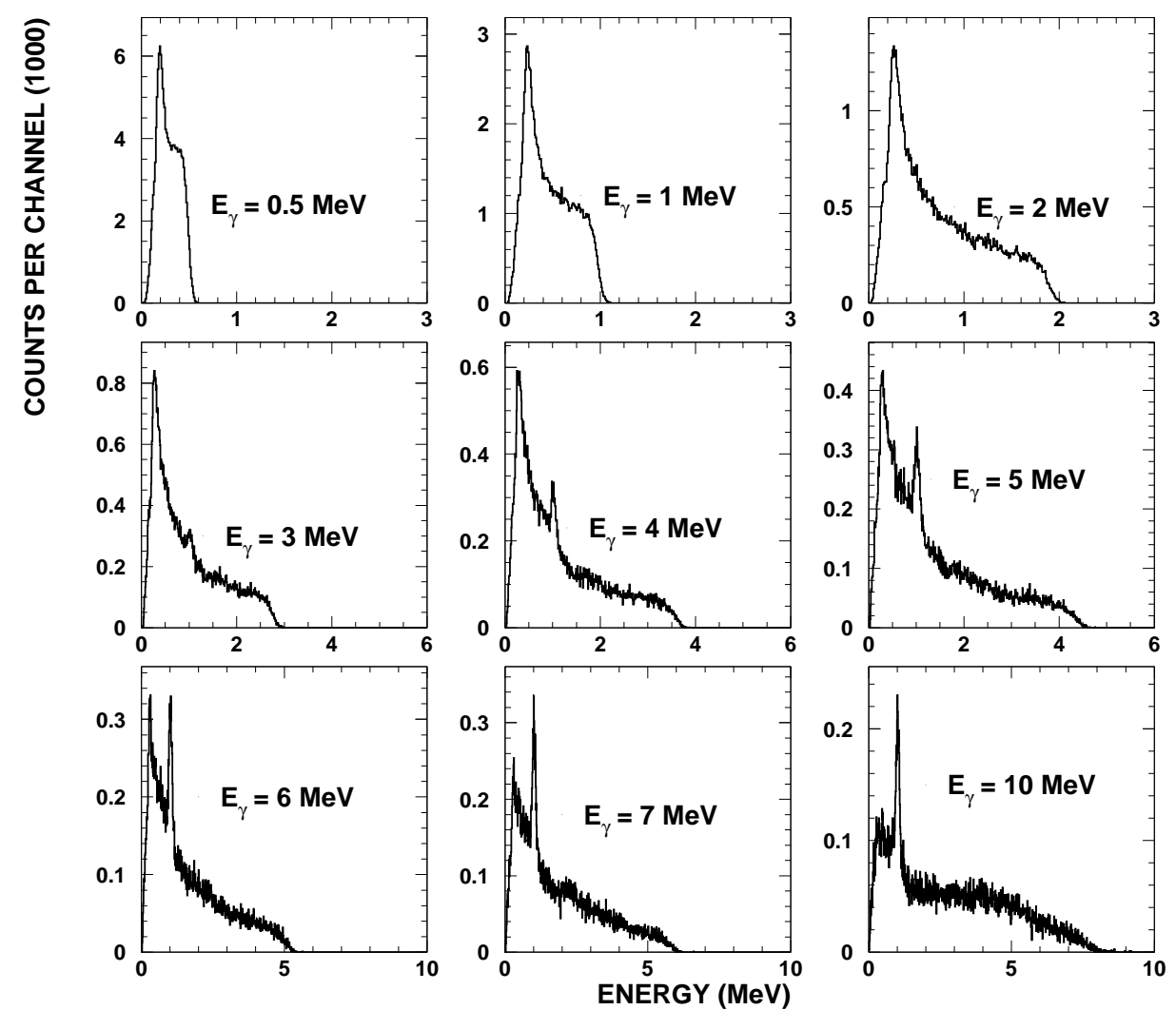

Fig. 13. Simulated total energy deposition in the $\mathrm{BaF}_{2}$-array for $10^{6} \gamma$-rays of different energies interacting with a carbon sample (10 mm thickness) in the center of the detector. The histogram binning is $10 \mathrm{keV} /$ channel. For more detailed information see Ref. [11].

More detailed investigations will be carried out with the completed array.

\section{Conclusions}

Modern neutron capture experiments require highly efficient detectors to enable the use of small samples, hence the investigation of rare or radioactive isotopes. In order to achieve a sufficient signal to background ratio, background suppression has to be emphasized and residual background components have to be well understood. During its first beam period the DANCE experiment concentrated on identifying, understanding and suppressing the applicable background components. This work was assisted by intensive Monte Carlo simulations.

As a result of these efforts the intrinsic $\alpha$-background can now be well separated from $\gamma$-events. In later stages these suppressed events will be used to automatically stabilize the high voltage of the PMT, which should in turn 

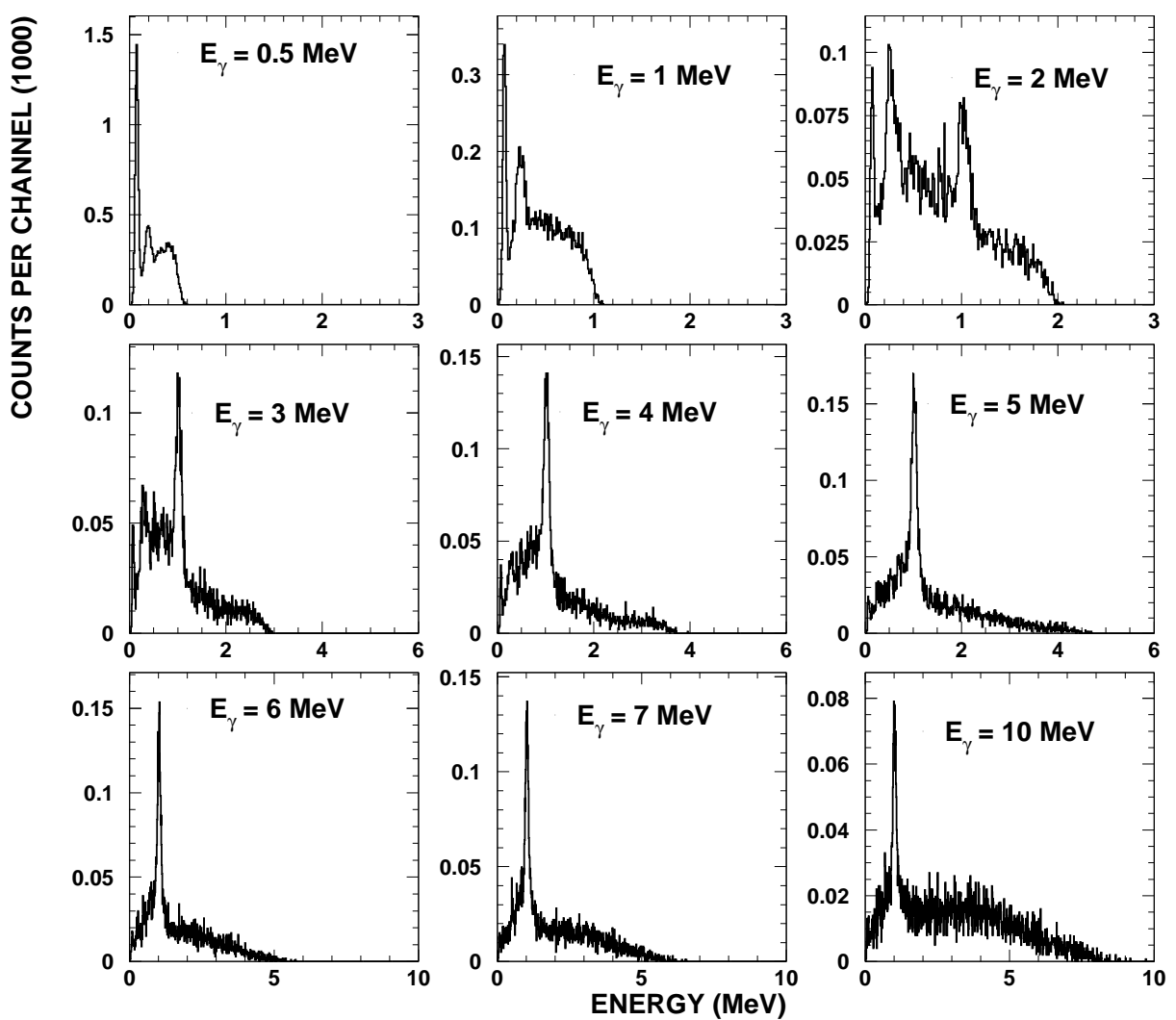

Fig. 14. Simulated total energy deposition in the $\mathrm{BaF}_{2}$-array for $10^{6} \gamma$-rays of different energies interacting with a lead sample $(0.2 \mathrm{~mm}$ thickness $)$ in the center of the detector. The histogram binning is $10 \mathrm{keV} /$ channel. For more detailed information see Ref. [11].

stabilize the energy calibration. Time-dependent background components induced by structural material in the beam line and shielding have been reduced by optimized choices of materials and dimensions. As expected from simulations, the installation of a ${ }^{6} \mathrm{LiH}$ absorber between sample and detector sphere reduces the background from scattered neutrons significantly. Although reducing the peak efficiency the use of this absorber shell clearly improves the signal to background ratio.

Gamma induced background will be further investigated, since it is shown to be an important component. Starting in Summer 2003 neutron capture measurements on a number of stable as well as unstable isotopes have been successfully carried out the DANCE. The analysis of these experiments is in progress. 


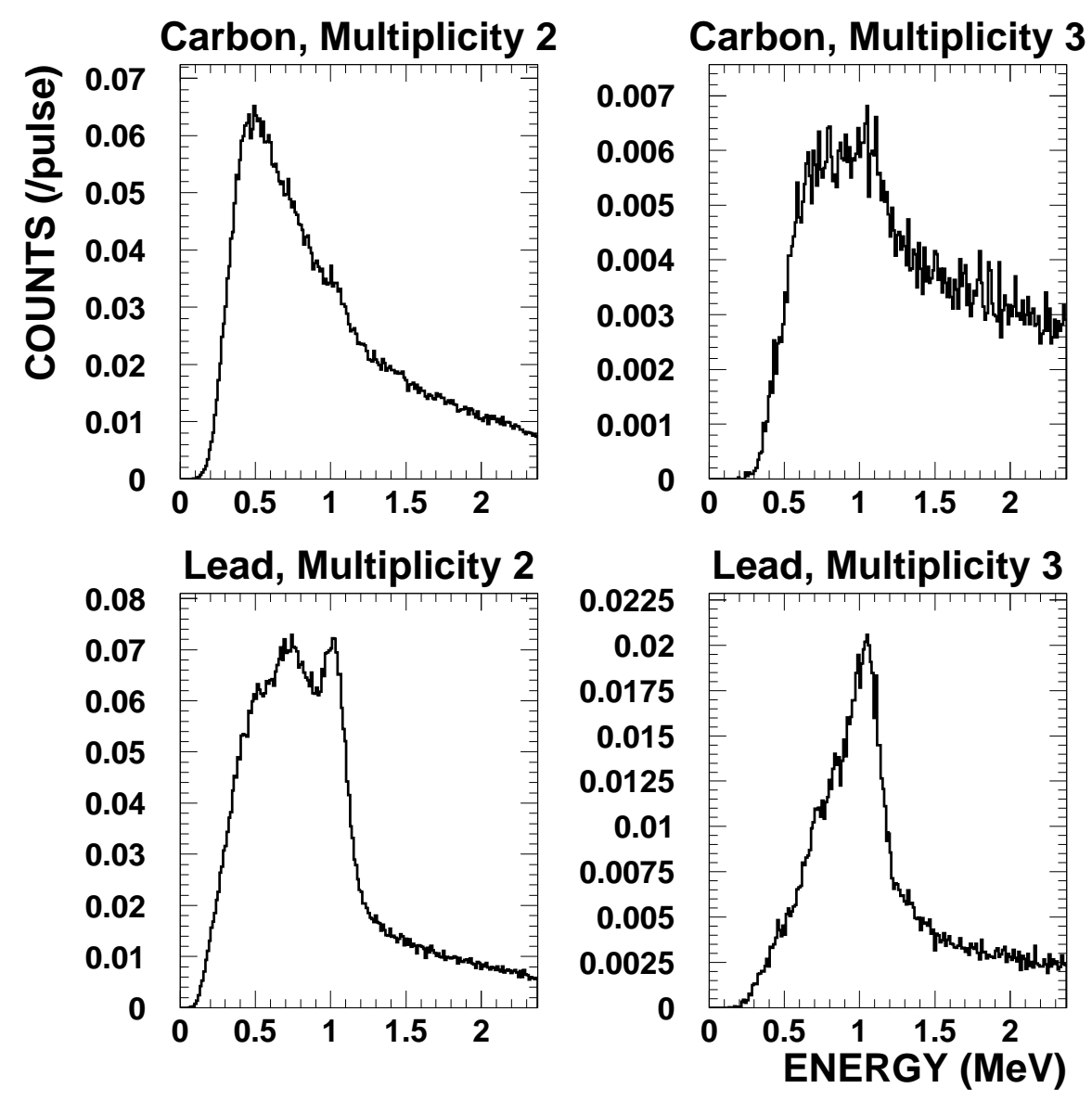

Fig. 15. Total energy spectra for a $\mathrm{C}$ and a $\mathrm{Pb}$ sample taken with the DANCE array. All measurements have been carried out for $0.5 \mathrm{~h}$ with a $1.3 \mathrm{~cm}$ polyethylene filter in the beam $10 \mathrm{~m}$ upstream of the sample. The top panels show the results for a $44.4 \mathrm{mg} / \mathrm{cm}^{2}$ carbon sample and the bottom for a $43.5 \mathrm{mg} / \mathrm{cm}^{2}$ lead sample. The left and right pictures correspond to crystal multiplicity 2 and 3, respectively. The TOF interval is the same as in Fig. 6. The histogram binning is $12 \mathrm{keV} /$ channel. One pulse corresponds to $100 \mu$ s looking time.

\section{Acknowledgements}

We would like to thank L.F. Hunt, E.P. Chamberlin, D.R. Harkleroad, N.F. Archuleta and D.M. Lujan for their invaluable help during the development and construction phase of DANCE. Special thanks also to M. Heil and F. Käppeler from FZK for countless clarifying discussions. This work has benefited from the use of the Los Alamos Neutron Science Center at the Los Alamos National Laboratory. This facility is funded by the US Department of Energy and operated by the University of California under Contract W-7405-ENG-36. The Colorado School of Mines group is funded via DOE grant: DE-FG02-93ER40789. 

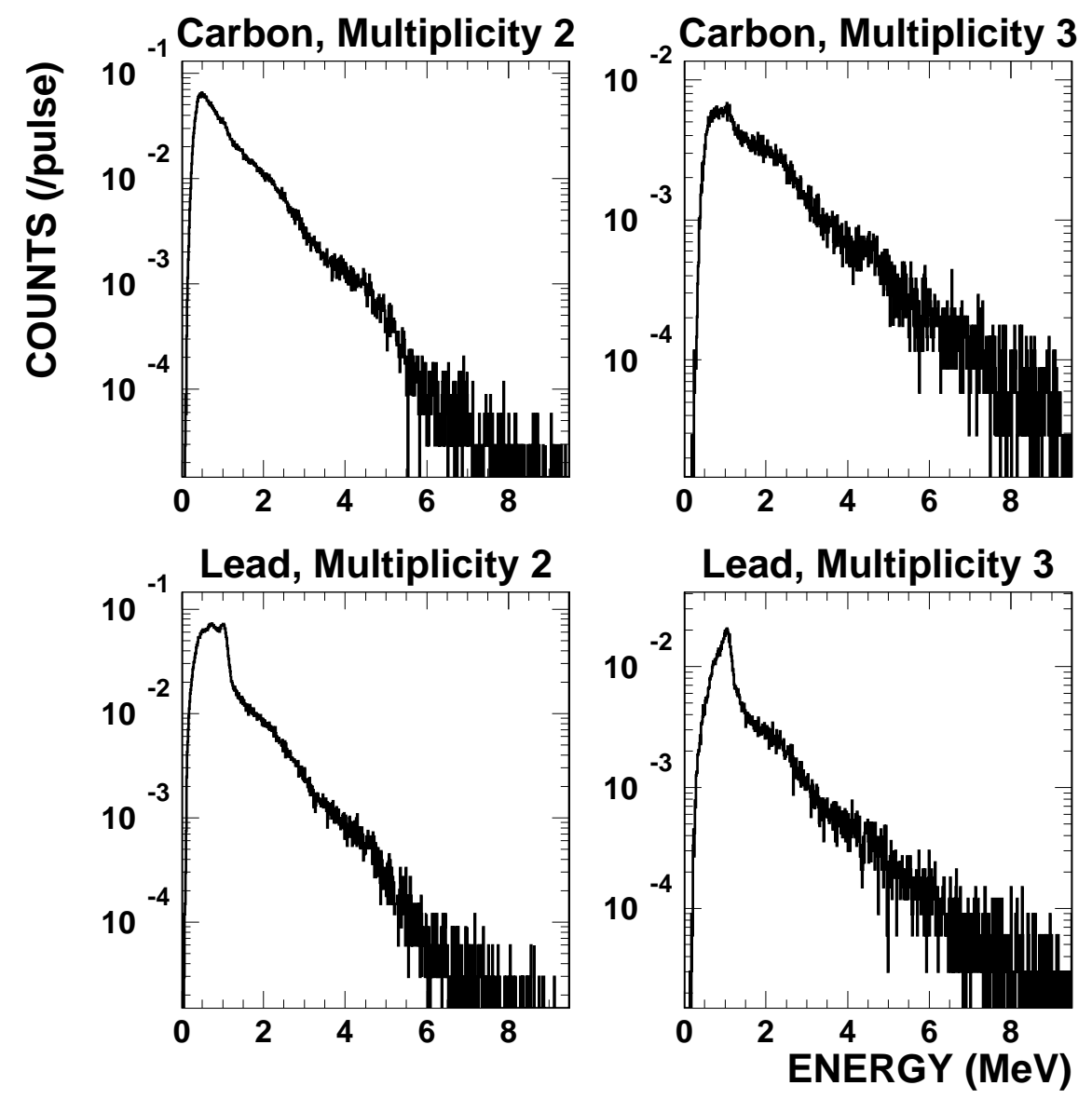

Fig. 16. The same data as in Fig. 15, but with different energy scale and logarithmic y-axis.

\section{References}

[1] P.W. Lisowski, C.D. Bowman, G.J. Russell, S.A. Wender, Nucl. Sci. Engineering 106 (1990) 208.

[2] K. Wisshak, K. Guber, F. Käppeler, J. Krisch, H. Müller, G. Rupp, F. Voss, Nucl. Instr. Meth. A 292 (1990) 595.

[3] M. Heil, R. Reifarth, M.M. Fowler, R.C. Haight, F. Käppeler, R.S. Rundberg, E.H. Seabury, J.L. Ullmann, J.B. Wilhelmy, K. Wisshak, F. Voss, Nucl. Instr. Meth. A 459 (2001) 229.

[4] D. Habs, F.S. Stephens, R.M. Diamond, Tech. rep., Report LBL-8945, Lawrence Berkeley Laboratory (1979).

[5] J. Apostolakis, CERN, Tech. rep., GEANT library, http://wwwinfo.cern.ch/asd/geant// (1993).

[6] C. Zeitnitz, T.A. Gabriel, Nucl. Instr. Meth. A 349 (1994) 106.

[7] E. Browne, R. Firestone, Table of Radioactive Isotopes, Wiley, New York, 1986.

[8] R.B. Firestone, Table of Isotopes, Wiley, New York, 1996. 
[9] R. Reifarth, M. Heil, F. Käppeler, F. Voss, K. Wisshak, R.C. Haight, M.R. Dragowsky, M.M. Fowler, R.S. Rundberg, J.L. Ullmann, J.B. Wilhelmy, E.H. Seabury, Tech. rep., Report LA-UR-01-4185, Los Alamos National Laboratory (2001).

[10] R. Reifarth, T.A. Bredeweg, J.C. Browne, E.-I. Esch, U. Greife, R.C. Haight, J.M. O’Donnell, A. Kronenberg, , R.S. Rundberg, J.L. Ullmann, D.J. Vieira, J.B. Wilhelmy, J.M. Wouters, Tech. rep., Report LAUR-03-5560, Los Alamos National Laboratory (2003).

[11] R. Reifarth, T.A. Bredeweg, J.C. Browne, E.-I. Esch, U. Greife, R.C. Haight, J.M. O’Donnell, A. Kronenberg, , R.S. Rundberg, J.L. Ullmann, D.J. Vieira, J.B. Wilhelmy, J.M. Wouters, Tech. rep., Report LAUR-03-5559, Los Alamos National Laboratory (2003).

[12] M. Uhl, J. Kopecky, in: F. Käppeler, K. Wisshak (Eds.), Nuclei in the Cosmos 1992, Institute of Physics Publishing, Bristol, 1993, 259. 